\title{
Tumefactive lesions in Retinal Vasculopathy with Cerebral Leukoencephalopathy and Systemic manifestations (RVCL-S) - a role for neuroinflammation?
}

Todd A. Hardy ${ }^{1,2}$, Stephanie Young ${ }^{3}$, Joanne S. Sy ${ }^{4}$, Alison F. Colley ${ }^{5}$, Gisela M. Terwindt $^{6}$, Michel D. Ferrari ${ }^{6}$, Michael W. Hayes ${ }^{1}$ and Suzanne Hodgkinson ${ }^{7}$

1. Department of Neurology, Concord Repatriation General Hospital, University of Sydney, NSW, Australia

2. Brain and Mind Centre, University of Sydney, NSW, Australia

3. Department of Ophthalmology, Concord Repatriation General Hospital, University of Sydney, NSW, Australia

4. Department of Anatomical Pathology, Concord Repatriation General Hospital, University of Sydney, NSW, Australia

5. Department of Clinical Genetics, Liverpool Hospital, Liverpool, NSW, Australia

6. Department of Neurology, Leiden University Medical Center, Leiden, The Netherlands.

7. Department of Neurology, Liverpool Hospital, University of NSW, Australia

Short title: RVCL-S and tumefactive lesions

Keywords: TREX1, HERNS, HVR, CRV, pseudotumour, V235fs, multiple sclerosis, vasculitis

\section{Word count: 998}

No. of references: 12

Author for correspondence: Todd A. Hardy

Address: Department of Neurology, Concord Repatriation General Hospital, Hospital Rd, Concord, NSW 2139

Tel No. +61297675000

Fax No. +61 297676991 
E-mail: thardy@med.usyd.edu.au

\section{Email addresses of co-authors:}

Todd A. Hardy: thardy@med.usyd.edu.au

Stephanie H. Young: stephanieyoung@bigpond.com.au

Joanne S. Sy: drjoannesy@gmail.com

Alison F. Colley: alison.colley@sswahs.nsw.gov.au

Gisela Terwindt: G.M.Terwindt@lumc.nl

Michel D. Ferrari: M.D.Ferrari@lumc.nl

Michael W. Hayes: Michael.Hayes@sswahs.nsw.gov.au

Suzanne Hodgkinson: s.hodgkinson@unsw.edu.au 
Retinal Vasculopathy with Cerebral Leukoencephalopathy and Systemic manifestations (RVCL-S) is a rare, autosomal dominant condition caused by mutations in the TREXI gene. ${ }^{1,2}$ Typically, vascular retinopathy becomes apparent in the fourth or fifth decade, soon followed by clinical manifestations of progressive focal and global brain disease. Brain imaging reveals rim-enhancing mass lesions and/or punctate hyperintense white matter lesions with or without nodular enhancement, often in combination with focal white matter calcifications. Many patients will also have, or develop, liver and kidney dysfunction, anaemia with or without gastrointestinal bleeding, hypertension, migraine, and mild Raynaud's phenomenon. ${ }^{2}$ We describe two brothers with genetically-proven RVCL-S due to a mutation in the TREX1 gene (3688_3689insG) encoding the mutant protein V235fs. The clinical history, radiology, treatment and outcomes for the two brothers are presented as online supplementary data (figure S1).

In Case 1, multiple sclerosis (MS) with tumefactive demyelinating lesions, cerebral vasculitis and neoplasm were all considered as diagnostic possibilities until the patient's brother also became symptomatic, indicating a possible genetic cause. The father of the two brothers was probably affected but confirmation is no longer possible. The consanguineous family history in our cases is unlikely to be significant as RVCL-S is an autosomal dominant rather than recessive condition. A recessive mutation in TREX1 causes Aicardi-Goutieres syndrome (AGS), which presents in the first year of life with acquired microcephaly, developmental delay, encephalopathy, spasticity and extrapyramidal signs. Like RVCL-S, AGS is notable for neuropathology demonstrating microangiopathy and microinfarction. 
The MRI features of RVCL-S include periventricular white matter T2 hyperintensities, calcifications and infarcts. ${ }^{2}$ Tumefactive lesions are found in approximately $50 \%$ of cases with a predilection for the frontoparietal lobes. ${ }^{3}$ When accompanied by smaller periventricular lesions these may be mistaken for tumefactive demyelination.

As in Case 1, neuropathology in patients with RVCL-S reveals areas of ischaemia and necrosis secondary to an occlusive endotheliopathy of small and medium-sized arteries (Fig. 1). ${ }^{2}$ Microvascular endothelial dysfunction also links clinical symptoms of Raynaud's phenomenon, migraine, and subcortical cognitive dysfunction and clinical signs of renal impairment, abnormal liver enzymes, gastrointestinal bleeding, anaemia and hypertension. ${ }^{2}$

In some lesional brain biopsies, modest inflammatory lymphocytic infiltrates are also seen. It has been proposed that these inflammatory cells are due to breach of the blood-brain-barrier in reaction to focal cerebral ischaemia. ${ }^{2}$ Another interpretation, however, would be that there is an inflammatory contribution to lesion pathogenesis. Indeed, a link between TREX1 mutations and immunity is now recognised. Certain mutations in the TREXI gene, which encodes the major mammalian 3'-5' DNA exonuclease, lead to cytosolic accumulation of nucleic acids that trigger an innate immune response by binding to cyclic GMP-AMP synthase (cGAS). This can lead to upregulation of type 1 interferon, and downstream autoimmunity similar to that induced by viral nucleic acid, ${ }^{4}$ although it has not been shown whether there is an upregulation of type 1 interferon in the plasma of patients with RVCL-S. In humans, $2-3 \%$ of patients with the immune-mediated condition systemic lupus erythematosis (SLE) have TREX1 mutations, and heterozygous mutations in TREX1 underlie the 
autoimmune condition familial chilblain lupus. As in SLE, AGS is associated with elevated CSF interferon-alpha and AGS patients may not only have typical lupus autoantibodies but also may manifest frank autoimmune disease. ${ }^{5}$ In Trex 1 null mice, an inflammatory myocarditis develops that is hypothesised to be due to build-up of retroelement complementary DNA. ${ }^{6}$ Other organs in these mice also demonstrate lymphocytic inflammatory infiltrates, although the brain is relatively spared. ${ }^{7}$

A role for neuroinflammation in lesion pathogenesis might help to explain the marked perilesional oedema which accompanies the tumefactive lesions, which is in excess of that seen in conventional ischaemic stroke. In addition, neuroinflammation may explain the tendency for RVCL-S lesions to relapse and remit as in MS. ${ }^{3}$ An overlap between microvascular ischaemia and neuroinflammation has been proposed in other immune-associated CNS conditions such as antiphospholipid syndrome and Susac's syndrome. $^{8}$

We reported that Case 1 developed lower limb macular rash. Another patient with RVCL-S also developed punctate skin lesions on the thighs ${ }^{9}$ and yet another was reported to have skin lesions. ${ }^{10}$ In the first of these cases, as in our case, pathology was consistent with "vasculitis". Hence, cutaneous manifestations may also form part of the broader clinical phenotype of RVCL-S. Further support comes from the observation that electron microscopy of a skin biopsy specimen in a patient with RVCL-S revealed abnormal multilaminated vascular basement membranes analogous to those seen in brain specimens. ${ }^{11}$

Case 1 improved transiently following corticosteroids when tumefactive lesions developed, presumably due to an effect on lesional inflammation and oedema, a 
phenomenon which has been noted in other cases. ${ }^{11,12}$ No treatment has been described, however, that can arrest or slow progression of RVCL-S. ${ }^{12}$ As RVCL-S mutations are likely to cause a toxic gain-of-function it seems unlikely that candidates tested in the Trex1-null mice (loss-of-function) will provide a future therapeutic strategy for patients with RVCL-S. ${ }^{4}$

Although our patient continued to relapse despite treatment with a number of immunotherapies, it is conceivable that targeted immune therapies could modify the disease course. We are not aware of any cases of RVCL-S treated with the alpha4integrin monoclonal antibody inhibitor, natalizumab, but potentially this could reduce lymphocyte tracking across the abnormal vascular endothelium in this condition and modify CNS lesion formation, as in relapsing-remitting multiple sclerosis.

Vodopivec and colleagues tried the JAK-1 inhibitor, tofacitinib in their RVCL-S patient with the rationale of it reducing intracellular interferon alpha, and coadministered it with maintenance dexamethasone, and hydroxychloroquine, which inhibits activation of Toll-like receptors 7 and 9 by exogenous nucleic acid ligands, and reduces interferon beta. ${ }^{12}$ Their patient developed numerous complications before the therapy could be adequately trialled, including infections attributed to prolonged corticosteroids, but they argue this targeted approach remains a potential therapeutic strategy in RVCL-S.

In conclusion, RVCL-S should be suspected in adult patients with unusual cerebral and retinal vascular pathology. We suggest that neuroinflammation could play a role in brain lesion formation in RVCL-S, the clinical phenotype of RVCL-S be broadened 
to include skin involvement, and that RVCL-S be considered a rare differential diagnosis of tumefactive demyelination.

\section{CONTRIBUTORSHIP STATEMENT}

TAH and MWH conceived the idea for the article and TAH, SY and MWH drafted the manuscript. SY supplied fluorescein angiogram images, and JSS the neuropathology. AFC reviewed the family history and suggested the diagnosis, and GMT and MDF performed the confirmatory DNA analysis. All authors revised the manuscript critically for important intellectual content, and gave final approval of the version to be published.

\section{DISCLOSURES}

No conflicts of interest are declared by the authors.

\section{ACKNOWLEDGEMENTS}

Nil

\section{FUNDING}

This work received no specific grant from any funding agency in the public, commercial or not-for-profit sectors. 


\section{References}

1. Richards A, van den Maagdenberg AM, Jen JC, et al. C-terminal truncations in human 3'-5' DNA exonuclease TREX1 cause autosomal dominant retinal vasculopathy with cerebral leukodystrophy. Nat Genet. 2007 Sep;39(9):1068-70.

2. Stam AH, Kothari PH, Shaikh A, et al. Retinal vasculopathy with cerebral leukoencephalopathy and systemic manifestations. Brain. 2016 Sep 6. pii: aww217. [Epub ahead of print].

3. Mateen FJ, Krecke K, Younge BR, Ford AL, Shaikh A, Kothari PH, Atkinson JP. Evolution of a tumor-like lesion in cerebroretinal vasculopathy and TREX1 mutation. Neurology. 2010 Sep 28;75(13):1211-3.

4. Gray EE, Treuting PM, Woodward JJ, Stetson DB. Cutting Edge: cGAS Is Required for Lethal Autoimmune Disease in the Trex1-Deficient Mouse Model of Aicardi-Goutières Syndrome. J Immunol. 2015 Sep 1;195(5):1939-43.

5. Olivieri I, Cattalini M, Tonduti D, La Piana R, Uggetti C, Galli J, Meini A, Tincani A, Moratto D, Fazzi E, Balottin U, Orcesi S. Dysregulation of the immune system in Aicardi-Goutières syndrome: another example in a TREX1-mutated patient. Lupus. 2013 Sep;22(10):1064-9.

6. Beck-Engeser GB, Eilat D, Wabl M. An autoimmune disease prevented by antiretroviral drugs. Retrovirology. 2011 Nov 8;8:91.

7. Pereira-Lopes S, Celhar T, Sans-Fons G, Serra M, Fairhurst AM, Lloberas J, Celada A. The Exonuclease Trex1 Restrains Macrophage Proinflammatory Activation. J Immunol. 2013 Dec 15;191(12):6128-35.

8. Hardy TA, O'Brien B, Gerbis N, Barnett MH, Reddel SW, Brewer J, Herkes GK, Silberstein P, Garsia RJ, Watson JD, Gupta R, Parratt JD, Buckland ME. Brain histopathology in three cases of Susac's syndrome: implications for lesion pathogenesis and treatment. J Neurol Neurosurg Psychiatry. 2015 May;86(5):582-4.

9. Gutmann DH, Fischbeck KH, Sergott RC. Hereditary retinal vasculopathy with cerebral white matter lesions. Am J Med Genet. 1989 Oct;34(2):217-20.

10. DiFrancesco JC, Novara F, Zuffardi O, Forlino A, Gioia R, Cossu F, Bolognesi M, Andreoni S, Saracchi E, Frigeni B, Stellato T, Tolnay M, Winkler DT, Remida P, Isimbaldi G, Ferrarese C. TREX1 C-terminal frameshift mutations in the systemic variant of retinal vasculopathy with cerebral leukodystrophy. Neurol Sci. 2015 Feb;36(2):323-30.

11. Jen J, Cohen AH, Yue Q, Stout JT, Vinters HV, Nelson S, Baloh RW. Hereditary endotheliopathy with retinopathy, nephropathy, and stroke (HERNS). Neurology. 1997 Nov;49(5):1322-30.

12. Vodopivec I, Oakley DH, Perugino CA, Venna N, Hedley-Whyte ET, Stone JH. A 44-year-old man with eye, kidney, and brain dysfunction. Ann Neurol. 2016 Apr;79(4):507-19. 
Figure 1 (A) Late phase fluorescein angiogram from Case 1 showing profound retinal ischaemia with capillary dropout, neovascularisation of the disc and elsewhere, and vitreous haemorrhage. (B) MRI brain of Case 1 showing fluid attenuated inversion recovery (FLAIR) and (C) post gadolinium T1 sequence and (D) diffusion-weighted sequence showing an enhancing tumefactive lesion (pseudotumor) in the right frontal lobe with surrounding oedema. Additional smaller periventricular enhancing lesions are also evident in the white matter of the left hemisphere. (E) Brain biopsy haematoxylin \& eosin stain from Case 1 showing perivascular lymphocytic cuffing (magnification x 600) with inset showing CD3+ lymphocytes. (F) Thick walled vessels within an area of brain lesion necrosis (magnification x 400). (G) Brain biopsy from Case 1 showing macrophages stained with CD68 with inset showing microglial cells stained for CD163. (H) Skin biopsy haematoxylin \& eosin stain from Case 1 (magnification x600) showing perivascular inflammatory cell infiltrate with leucocytoclasis (fragmented white blood cells) and extravasation of red blood vessels. The features are suggestive of a leucocytoclastic vasculitis. (I) MRI brain of Case 2 with axial FLAIR sequence showing a pseudotumour in the right frontal lobe with surrounding oedema and midline shift with marked oedema. (J) Brain biopsy haematoxylin \& eosin stain from Case 2 showing perivascular lymphocytic cuffing (magnification x 600) with inset showing CD3+ lymphocytes. (K) Thick walled vessels within an area of brain lesion necrosis (magnification $\mathrm{x} 400$ ). (G) Brain biopsy from Case 2 showing macrophages stained with CD68 with inset showing microglial cells stained for CD163. 


\section{CASE REPORTS}

\section{Case 1}

A 35-year-old man presented to his Ophthalmologist in March 2001 complaining of floaters in the left eye with reduced vision of 6/36. Examination revealed vitreous haemorrhage and retinal ischaemia with areas of non-perfusion and neovascularisation confirmed by fluorescein angiography (FA; Fig. 1A). Routine blood tests, serum vasculitis screen and CSF protein and cell count were normal. MRI brain revealed small foci of T2 hyperintensity in the left frontal lobe with a solitary punctate focus of gadolinium enhancement. Neuropsychological testing revealed mild cognitive slowing. Single photon emission computed tomography (SPECT) showed a generalized patchy pattern of cerebral cortical perfusion. He was diagnosed with a "cerebro-retinal vasculitis" and treated empirically with high dose prednisolone followed by intravenous (IV) cyclophosphamide monthly for three courses together with pan-retinal laser photocoagulation. His vision improved to $6 / 6$ although a nasal hemi-field defect in his left eye persisted.

At 12-month follow-up, he complained of headache, deteriorating vision in the left eye and had focal left sided neurological signs with increased tone, bradykinesia and hyperreflexia in the left upper limb. Cognitively, he was slower and comprehension was impaired. CSF showed an elevated protein of $0.65 \mathrm{~g} / \mathrm{L}$ and repeat FA indicated active retinal vasculopathy. MRI brain showed a gadolinium-enhancing tumefactive lesion $(2 \mathrm{~cm})$ with surrounding oedema in the right frontal lobe and multiple periventricular gadolinium-enhancing lesions bilaterally (Fig. 1B-D). 
Brain biopsy in April 2002 revealed cerebral white matter necrosis with reactive astrocytosis in adjacent brain parenchyma, and perivascular lymphocytic cuffing (Fig. 1E-G). Some vessels were tortuous with thick hyalinised walls (Fig. 1F). Subsequently, he developed six macular lesions on his left calf, and biopsy showed fibrinoid necrosis as well as early dermal degenerative changes and ulceration consistent with leukocytoclastic vasculitis (Fig. 1H). Follow-up MRI brain showed waxing and waning in the size of the white matter lesions.

By mid-2003 acuity in the right eye had worsened due to further retinal ischaemia and neovascularisation. In his left eye, acuity was count fingers and there was iris rubeosis secondary to retinal ischaemia with increased intraocular pressure. MRI brain in December 2003 showed multiple large, confluent periventricular gadolinium-enhancing lesions in the deep white matter of the frontal lobes. By February 2004 he required hospitalisation because of marked dysphagia with prominent dysarthria, left-sided weakness, ataxia and falls. By September 2004 he was confined to a wheelchair and his speech was almost unintelligible. Throughout his illness, due to persistent uncertainty regarding the diagnosis, including the possibility this might have been an atypical form of multiple sclerosis with recurrent tumefactive demyelination, he was variously given courses of intravenous and oral corticosteroids, beta-interferon, glatiramer acetate, cyclophosphamide and plasmapheresis all of which failed to prevent further relapses. He continued to deteriorate and died in January 2008. The course of his worsening clinical disability is shown in Fig. S1.

\section{Case 2}

The 41-year-old brother of Case 1 presented in 2004 with a one-week history of morning headaches associated with nausea, confusion and drowsiness. MRI revealed a 
large, deep, right frontal ring-enhancing mass with oedema and mass effect (Fig. 1I). A brain tumour was suspected but biopsy of the lesion showed a microvascular angiopathic process with coagulative and fibrinoid necrosis within and around the lesion and scattered neutrophils, mononuclear cells and histiocytes (Fig. 1J-L). Rare degenerate vessels had a hyaline thickened appearance suggesting intimal proliferation (Fig 1K).

The only abnormalities on extensive serum testing were elevated serum gammaglutamyltransferase of 239 units/L and alkaline phosphatase of 114 units/L. FA showed asymptomatic retinal capillary non-perfusion consistent with early retinal disease. Follow-up MRI six months later showed new bifrontal white matter hyperintensities (anterior corpus callosum and deep white matter of the left frontal lobe).

In view of both brothers' cerebroretinal pathology, a more detailed family history was taken. Their father had died in a nursing home at the age of 65 following a slowly progressive, stepwise illness characterised by worsening mobility and cognition which commenced in his 50s and was attributed to multiple strokes. Both he and his wife were of Calabrian Italian descent and consanguineous in that they were children of identical twin brothers. DNA was obtained from Case 1 and 2 and subsequent analysis showed a mutation in the TREX1 gene (3688_3689insG) encoding the mutant protein V235fs and the diagnosis of RVCL-S was confirmed (see Family 5 in Richard et al. 2017 and Stam et al. 2016). ${ }^{1,2}$

Case 2 was managed conservatively with an emphasis on symptom control and rehabilitation between attacks of neurological dysfunction. At the present time, thirteen 
years since presentation, he is living in a nursing home, visually impaired, bedbound, aphasic and prone to status epilepticus (Fig. S1). 
Supplementary Fig. 1 The clinical disability of the two cases over time as per their Modified Rankin Scale (MRS) score. Case 1 is denoted by an X and Case 2 is denoted by a $\boldsymbol{\Lambda}$. MRS $0=$ no symptoms; MRS $1=$ no significant disability, able to carry out all usual activities, despite some symptoms; MRS 2 = slight disability, able to look after own affairs without assistance, but unable to carry out all previous activities; MRS $3=$ moderate disability, requires some help, but able to walk unassisted; MRS $4=$ moderately severe disability, unable to attend to own bodily needs without assistance, and unable to walk unassisted; MRS 5 = severe disability, requires constant nursing care and attention, bedridden, incontinent; MRS $6=$ dead. 


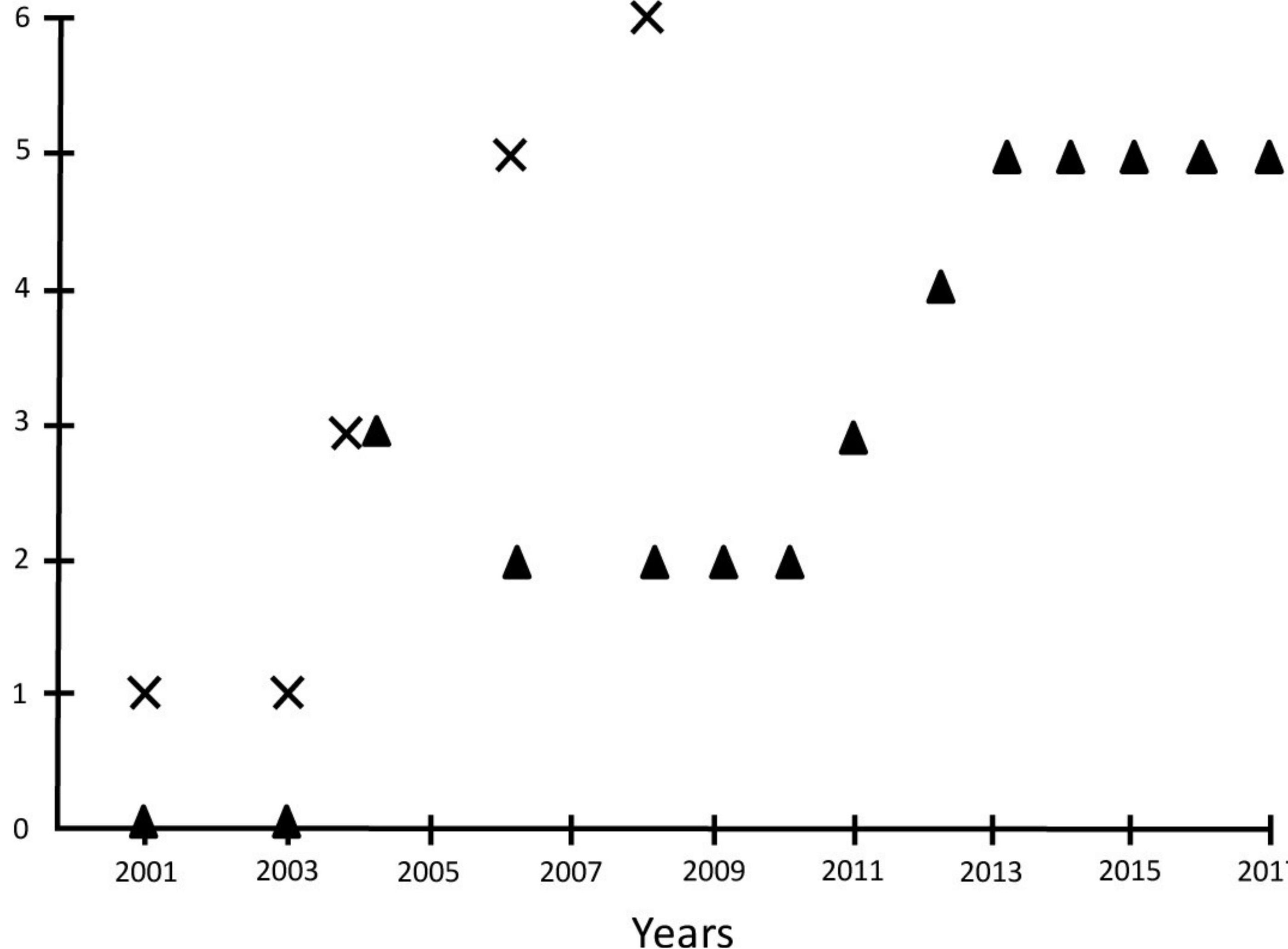

